\title{
Polarizable QM/MM multi-configuration self- consistent field approach with state-specific corrections: environment effects on cytosine absorption spectrum
}

\author{
Quansong Li, ${ }^{a}$ Benedetta Mennucci, ${ }^{b}$ Michael A. Robb,${ }^{c}$ Lluís Blancafort ${ }^{* d}$ \\ Carles Curutchet ${ }^{*}$
}

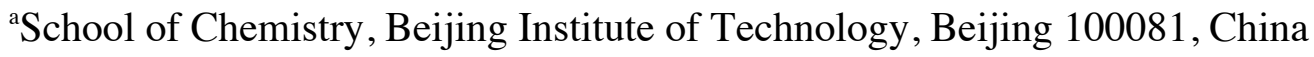

${ }^{b}$ Dipartimento di Chimica e Chimica Industriale, University of Pisa, Via G. Moruzzi, 56124 Pisa, Italy

'Department of Chemistry, Imperial College London, London SW7 2AZ, United Kingdom

Institut de Química Computacional i Catàlisi and Departament de Química, Universitat de Girona, Campus Montilivi, 17071 Girona, Spain

eDepartament de Fisicoquímica, Facultat de Farmàcia, Universitat de Barcelona, Av. Joan XXIII s/n, 08028 Barcelona, Spain

*To whom correspondence should be addressed. E-mail: carles.curutchet@ub.edu and lluis.blancafort@udg.edu

\section{ABSTRACT}

We present the formulation and implementation of a polarizable quantum mechanics/molecular mechanics (QM/MM) strategy to describe environment effects in multi-configuration self-consistent field calculations. The strategy is applied to the calculation of the vertical absorption spectrum of cytosine in water. In our approach, mutual polarization of the solute and the solvent is solved self-consistently at the complete-active-space self-consistent-field (CASSCF) level, and the resulting set of charges and dipoles is used to calculate vertical excitation energies using the completeactive-space second-order perturbative (CASPT2) approach and its multi-state (MSCASPT2) variant. In order to treat multiple excited states, we converge the solvent polarization with respect to the state-averaged density of the solute. In order to obtain the final energies, however, we introduce a state-specific correction, where the solvent polarization is recomputed with the density of each state, and demonstrate that this 
correction brings the excitation energies closer to the values obtained with stateoptimized orbitals. Comparison with PCM and non-polarizable QM/MM calculations shows the importance of specific solute-solvent interactions and environment polarization in describing experiments. Overall, the calculated excitations for the $\pi \rightarrow \pi^{*}$ states in water show good agreement with the experimental spectrum, whereas the $n \rightarrow \pi^{*}$ appear at energies above $6 \mathrm{eV}$, approximately $1 \mathrm{eV}$ higher than in the gas phase. Beyond solvents, the new method will allow studying the impact of heterogeneous biological environments in multiple excited states, as well as the treatment of multichromophoric systems where charge transfer and exciton states play important roles.

\section{Introduction}

Environment effects play a key role in photochemistry and photophysics. ${ }^{1-4}$ The change in chromophore-environment interaction energy concomitant to a change in electronic state can lead, for instance, to significant shifts in the energies of excited states in vacuum compared to a condensed phase. This can modulate the potential energy surfaces and the internal conversion pathways available for a molecule to dissipate the energy after photon absorption. In complex systems where several chromophores come into play, the environment can modulate excited state dynamics in a variety of ways, for example by stabilizing charge transfer states or by modulating the electronic coupling promoting photoinduced charge and energy transfer processes. This complexity is exemplified by DNA photoprotection mechanisms, where internal conversion competes with charge and energy transfer processes highly sensitive to the surrounding environment. ${ }^{5-6}$ Several fluorescent probes are also used to reveal a variety of biochemical and cellular processes with unprecedented detail, and in such cases a good understanding of the underlying photophysics is critical in the interpretation and design of the experiments. ${ }^{7}$

This importance has led to continuous efforts to develop and improve the models that account for the environment in excited state quantum chemical calculations., 4 These models typically resort to hybrid models in which the quantum-mechanical (QM) 
description of the chromophore is combined with a classical description of the environment through continuum or molecular mechanics (QM/MM) models. Continuum models, where the environment is modeled as a homogeneous dielectric medium, are very popular nowadays due to their efficiency and remarkable accuracy, especially for homogeneous environments (solvents) where no strong specific solutesolvent interactions are present. When strong interactions like persistent hydrogen bonds are present, or in complex systems where the environment is heterogeneous, like in biological matrices, $\mathrm{QM} / \mathrm{MM}$ models are instead expected to give a better description as they explicitly account for the structural details of the surrounding molecules. This advantage however generally implies a larger computational cost associated to QM/MM compared to continuum models, because predicted properties need to be ensembleaveraged, typically over a set of structures extracted from a molecular dynamics (MD) simulation. Another problem with QM/MM concerns non-equilibrium solvation effects arising from the inertial (slow) component of the polarization of the environment. These effects can be incorporated into continuum solvation models in a straightforward way by considering different dielectric constants for the fast and slow components of the reaction field, but they are not properly accounted for in QM/MM because the MM description is limited to point charges based on standard non-polarizable force fields. To overcome this limitation, several groups have developed QM/MM methods based on polarizable force fields, ${ }^{8-18}$ an idea already introduced in the seminal QM/MM paper by Warshel and Levitt. ${ }^{19}$ Such approaches solve for mutual polarization effects among the solute and the solvent until self-consistency. In particular, some of the present authors have recently developed a polarizable $\mathrm{QM} / \mathrm{MM}$ (QM/MMpol) method to describe excited states and energy transfer in condensed phase based on a linear response approach, which can be applied at the configuration-interaction of single excitations (CIS), ${ }^{15}, 20$ Zerner's semiempirical intermediate neglect of differential overlap (ZINDO), ${ }^{21}$ or time-dependent density functional theory (TD-DFT) levels of theory. ${ }^{15}$ Similar implementations for TD-DFT have also been described. ${ }^{22-23}$

In this work, we extend the QM/MMPol methodology to the complete-activespace self-consistent-field (CASSCF) method.$^{24}$ This is one of the reference methods for excited states, specially for the study of potential energy surfaces, in combination with the complete-active-space second-order perturbative approach CASPT2 ${ }^{25}$ and its multistate variant MS-CASPT2. ${ }^{26}$ In our implementation, the solvent potential (electrostatic 
plus polarization) is obtained at the CASSCF level as an external perturbation. A similar implementation has been described recently where the solvent response is calculated with respect to a specific electronic state..$^{27}$ In contrast, our method is based on stateaveraged CASSCF and allows for the treatment of several excited states. During the CASSCF procedure, the solvent polarization response is converged with respect to the state-averaged density of the solute. This is complemented with a simple yet efficient correction to estimate the state-specific interaction energy with the solvent. Once the polarization and the state-averaged density are converged, the state-specific polarization is recomputed for each transition to correct for the final energies. Finally, the stateaveraged polarization is used to calculate the CASPT2 and MS-CASPT2 energies.

We apply our novel method to model the excitation spectrum of the canonical cytosine keto $\mathrm{N} 1 \mathrm{H}$ tautomer (see Figure 1), which is known to be the main one in aqueous solution. ${ }^{28}$ This subject is of great interest in the context of the photophysics of the DNA nucleobases. ${ }^{29}$ Cytosine, in particular, is a challenging test system because it presents several low-lying states of $n \rightarrow \pi^{*}$ and $\pi \rightarrow \pi^{*}$ character that behave differently upon solvation. In the gas phase, the vertical excitation energy of the lowest $\pi \rightarrow \pi^{*}$ transition, estimated from electron energy loss spectroscopic measurements, is $4.65 \mathrm{eV}$ or $266 \mathrm{~nm}^{30}$ Theoretically, most methods including CCSD(T), ${ }^{31}$ MS-CASPT2, ${ }^{32}$ CASPT $2,^{33}$ MR-CI ${ }^{34}$ DFT/MR-CI,${ }^{35}$ TD-DFT ${ }^{36}$ and ADC $(2)^{37}$ estimate that the $\pi \rightarrow \pi^{*}$ transition is the lowest vertical excitation $\left(S_{1}\right)$ in the gas phase (4.57-5.14 eV), with the lowest $n \rightarrow \pi^{*}$ transition appearing as $\mathrm{S}_{2}(4.76-5.54 \mathrm{eV})$. Depending on the method, $\mathrm{S}_{2}$ is assigned to an excitation coming from the oxygen or nitrogen lone pair. The vertical excitation spectrum in water has been calculated theoretically with various multireference methods using the $\mathrm{QM} / \mathrm{MM}$ and continuum approaches, ${ }^{38-39}$ and the calculated solvatochromic shifts are approximately $0.2 \mathrm{eV}$, for the lowest $\pi \rightarrow \pi^{*}$ transition, and $0.6-0.8 \mathrm{eV}$ for the two lowest $n \rightarrow \pi^{*}$ transitions. More recently, the spectrum of cytosine in water has been calculated taking the internal degrees of freedom of the molecule into account and using a continuum approach for the solvent, ${ }^{40}$ and the calculated shifts of the band maxima with respect to the vertical gas phase excitations are $0.4 \mathrm{eV}$, for the lowest $\pi \rightarrow \pi^{*}$ transition, and $0.4-0.6 \mathrm{eV}$ for the two lowest $n \rightarrow \pi^{*}$ transitions. 
The benchmark for our study in solution is the experimental spectrum at wave lengths higher than $190 \mathrm{~nm} .{ }^{41}$ This part of the spectrum has been fitted to four bands at 264, 233, 214 and $195 \mathrm{~nm}$, which are labeled I - IV in order of increasing energy. The relative intensity of the bands follows the order IV > III > I > II. A complete simulation of the spectrum is out of the scope of the paper, since it would require sampling the internal degrees of freedom of cytosine and including the eventual contribution of other, minor tautomers. ${ }^{40}$ Here we have computed the vertical excitations and compared them with the experimental bands. We have obtained the energies of eleven states, four $n \rightarrow \pi^{*}$ and seven $\pi \rightarrow \pi^{*}$ states. The polarization contribution to the solvent shift varies among the different states, leading to different relative energies. The results obtained with the proposed QM/MMpol scheme are also compared with calculations based on the Polarizable Continuum Model (PCM) ${ }^{42}$ and QM/MM without polarization. Comparison of the MS-CASPT2 vertical excitations with the maxima of the experimental bands indicates a continuous improvement of the predicted energy values for the first four $\pi \rightarrow \pi^{*}$ states when passing from PCM to QM/MM and QM/MMpol. The average difference between the two is reduced from 0.39 to 0.34 and 0.31 . This illustrates the importance of polarization effects. The final values are also in good agreement with the experimental results, specially if one takes into account that the vertical excitations tend to overestimate the experimental band maxima by $0.1-0.3 \mathrm{eV} .{ }^{43}$ In addition, the relative intensity of the four bands is qualitatively recovered when polarization effects are explicitly accounted for. For the $n \rightarrow \pi^{*}$ states, the blue shifts are of the order of $0.8-1.0$ $\mathrm{eV}$, and this results in vertical excitations of approximately $6 \mathrm{eV}$. Overall, the obtained results indicate that our method is a valuable tool to explore environment effects on multiple excited states.

\section{Methods}

\subsection{MC-SCF polarizable QM/MM model}

In this work we extend the polarizable QM/MM method previously developed for the treatment of excited states and electronic energy transfer at the CIS, ZINDO and TD-DFT levels to an MCSCF description of the excited states. ${ }^{15,44}$ This method explicitly accounts for environment polarization effects based on the induced point 
dipole model. Thus, MM sites are assigned an atomic partial charge and an isotropic atomic polarizability. Our extension is based on the MCSCF code implemented in the Gaussian software and in some aspects parallels the implementation of the PCM method. ${ }^{45}$ The Hamiltonian of the solute is modified by introducing a term accounting for the interaction between the QM and MM regions and a term describing the energy of the MM system. This leads to the following effective Hamiltonian:

$$
\hat{H}_{\mathrm{eff}}|\Psi\rangle=\left(\hat{H}_{0}+\hat{H}_{\mathrm{QM} / \mathrm{MM}}+\hat{H}_{\mathrm{MM}}\right)|\Psi\rangle=E|\Psi\rangle
$$

In Eq. (1), $\hat{H}_{0}$ is the Hamiltonian of the isolated QM system, the operator $\hat{H}_{\mathrm{QM} / \mathrm{MM}}$ introduces the interaction between the QM and the MM regions, and the operator $\hat{H}_{\mathrm{MM}}$ describes the internal energy of the MM region. The latter terms can be dissected into electrostatic and polarization terms:

$$
\begin{aligned}
& \hat{H}_{\mathrm{QM} / \mathrm{MM}}=\hat{H}_{Q M / M M}^{e l e}+\hat{H}_{Q M / M M}^{p o l} \\
& \hat{H}_{\mathrm{MM}}=\hat{H}_{M M}^{e l e}+\hat{H}_{M M}^{p o l}
\end{aligned}
$$

where $\hat{H}_{Q M / M M}^{e l e}$ and $\hat{H}_{Q M / M M}^{\text {pol }}$ describe the interaction between the QM system and the MM point charges and induced dipole moments, respectively, and $\hat{H}_{M M}^{e l e}$ and $\hat{H}_{M M}^{\text {pol }}$ contain the electrostatic self-energy of the MM charges and the polarization interaction between such charges and the induced dipoles. We note here that the $\hat{H}_{M M}^{e l e}$ term represents a constant energetic quantity, while the $\hat{H}_{M M}^{\text {pol }}$ contribution depends on the QM wavefunction through the induced dipoles. Empirical dispersion-repulsion terms often included in QM/MM models through empirical Lennard-Jones potentials are neglected here, given that they would not affect the properties of the wavefunction or the transition energies among excited states. By adopting this effective Hamiltonian, mutual polarization between the $\mathrm{QM}$ and the MM region is solved during the SCF procedure, through the induced dipoles that change at each cycle according to the change of the wavefunction. At each SCF iteration, mutual polarization between the dipoles is solved iteratively. This could also be achieved using a matrix inversion approach, but it would involve a larger computational cost. ${ }^{15}$ 
When an expansion of the wavefuntion over a finite basis set is introduced, the QM$\mathrm{MM}$ interacting operator can be split in the following electrostatic and polarization oneand two-electron terms:

$$
\begin{aligned}
& h_{\lambda v}^{e l e}=\sum_{i}^{M} q_{i} V_{\lambda v}\left(r_{i}\right)=-\sum_{i}^{M} q_{i}\left\langle\lambda \mid \frac{1}{\left|\overrightarrow{r_{i}-\vec{r} \mid}\right|}\right\rangle \\
& h_{\lambda v}^{p o l}=-\sum_{i}^{N} \vec{\mu}_{i}^{n} \vec{E}_{\lambda v}\left(r_{i}\right)=\sum_{i}^{N} \vec{\mu}_{i}^{n}\left\langle\lambda\left|\frac{\left(\vec{r}_{i}-\vec{r}\right)}{\left|\vec{r}_{i}-\vec{r}\right|^{3}}\right| v\right\rangle \\
& X_{\lambda v}^{p o l}=-\sum_{i}^{N} \vec{\mu}_{i}^{e} \vec{E}_{\lambda v}\left(r_{i}\right)=\sum_{i}^{N} \vec{\mu}_{i}^{e}\left\langle\lambda\left|\frac{\left(\vec{r}_{i}-\vec{r}\right)}{\left|\vec{r}_{i}-\vec{r}\right|^{3}}\right| v\right\rangle
\end{aligned}
$$

where $V_{\lambda v}$ and $\vec{E}_{\lambda v}$ indicate the electrostatic potential and electric field integrals, $q_{i}$ and $\mu_{i}$ the MM point charges and induced dipoles, and $N$ and $M$ the total number of charges and polarizable MM sites. In our implementation, induced dipoles are dissected into a nuclear (n) and an electronic (e) component, $\vec{\mu}_{i}^{n}=\alpha_{i} \vec{E}_{i}\left(Z_{n}, q_{m}, \vec{\mu}_{j \neq i}^{n}\right)$ and $\vec{\mu}_{i}^{e}=\alpha_{i} \vec{E}_{i}\left(\rho_{e}, \vec{\mu}_{j \neq i}^{e}\right)$, which depend on the nuclei and MM charges and on the electrons, respectively.

The final QM/MM and MM contributions to the energy can be expressed as:

$$
\begin{aligned}
& E_{Q M / M M+M M}=E^{e l e}+E^{p o l} \\
& E^{e l e}=\operatorname{tr} \mathbf{P} \mathbf{h}^{\text {ele }}+U_{n}^{\text {ele }}+U_{q}^{\text {ele }} \\
& E^{p o l}=\operatorname{tr} \mathbf{P} \mathbf{h}^{\text {pol }}+\frac{1}{2} \operatorname{tr} \mathbf{P} X^{p o l}(\mathbf{P})+\frac{1}{2} U_{n n}^{p o l}+\frac{1}{2} U_{q n}^{p o l}
\end{aligned}
$$

where $\mathbf{P}$ represents the one-electron density matrix on the chosen basis set, $U_{n}^{\text {ele }}$ describes the interaction between charges and nuclei, $U_{q}^{\text {ele }}$ the self-energy of the charges, and $U_{q n}^{p o l}$ and $U_{n n}^{p o l}$ the interaction between nuclear induced dipoles and nuclei and charges, respectively. 
In our QM/MMpol scheme we use the state-averaged (SA) density matrix because MCSCF calculations with state-optimized densities for excited states higher than $S_{1}$ suffer from root-flipping problems and do not converge. In order to estimate the energy corresponding to the proper state-specific polarization of the environment, at convergence we recompute the electron induced dipoles $\vec{\mu}_{i}^{e}$ in terms of the density of each state $\left(\mathbf{P}_{\text {state }}\right)$ and we introduce an energy correction defined as the difference between the energies recomputed with the two sets of dipoles, according to Equations (8) - (10):

$$
\begin{gathered}
E_{\text {corr }}=E_{Q M / M M+M M}\left(\mathbf{P}_{\text {state }}, \vec{\mu}_{i}^{e}\left(\mathbf{P}_{\text {state }}\right)\right)-E_{Q M / M M+M M}\left(\mathbf{P}_{\text {state }}, \vec{\mu}_{i}^{e}\left(\mathbf{P}_{S A}\right)\right) \\
\Delta E_{\text {corr }}=E_{\text {corr }}\left(S_{n}\right)-E_{\text {corr }}\left(S_{0}\right) \\
\Delta E_{S S}=\Delta E_{S A}+\Delta E_{\text {corr }}
\end{gathered}
$$

In the following, we present two sets of QM/MMpol data, those obtained with stateaveraged densities and the corresponding dipoles, $\Delta E_{S A}$, and those including the statespecific correction, $\Delta E_{S S}$. In addition, we have also implemented the QM/MMpol method at the CASSCF level with state-optimized orbitals, thus avoiding the stateaverage procedure, and we indicate this data by $\Delta E_{S O}$. In this case, the solvent polarization is self-consistently solved from the state-optimized density of the state of interest. Although this approach is only practical for the lowest excited state because of root flipping, it allows us to compare the shifts obtained with the state-specific correction to those obtained using state-optimized orbitals for $S_{1}$.

Finally, we recall that the state-specific correction is obtained at the CASSCF level, and then used to correct CASSCF, CASPT2 and MS-CASPT2 energies.

\subsection{Molecular dynamics simulations}

The structure of the cytosine keto $\mathrm{N} 1 \mathrm{H}$ tautomer was initially optimized at the MP2 level with the 6-311G(d,p) basis set, and then solvated in a box containing 1482 water molecules (buffer zone of $15 \AA$ ) using the Leap module of the Amber9 software. ${ }^{46}$ Water and cytosine were described using the TIP3P parameters ${ }^{47}$ and the ff99 force field, ${ }^{48}$ respectively, and the cytosine geometry was kept frozen during the MD simulations. The system was initially thermalized from 100 to $298 \mathrm{~K}$ during $100 \mathrm{ps}$ at 
constant volume, and then equilibrated for an additional 100 ps at constant pressure (1 atom) and temperature (298 K) MD using standard coupling schemes. The simulation was then extended $40 \mathrm{~ns}$ for production purposes. Simulations were performed using the Amber9 software using an integration time step of 1 fs, periodic boundary conditions, the Particle Mesh Ewald approach to deal with long-range electrostatics, and a nonbonded cutoff equal to $10 \AA$. QM/MM calculations were then performed extracting 101 structures each 200 ps over the last 20 ns.

\subsection{Quantum-chemical calculations}

CASSCF calculations were performed with an active space of 14 electrons in 10 orbitals and Dunning's cc-pVTZ basis set. Initial CASSCF calculations using the PCM and the novel implementation of the QM/MMpol model were performed using a locally modified version of the Gaussian09 code. ${ }^{49}$ For PCM calculations, a calculation on the ground-state was used to obtain the inertial reaction field equilibrated to the ground state, and this was later included in a non-equilibrium PCM calculation state-averaged over the first 12 states, where only the dynamical part of the reaction field was allowed to adapt to the SA density. QM/MMpol calculations were similarly performed adapting the solvent polarization response to the SA density, and the final energies were corrected by the solvent state-specific correction described in the section 2.1. QM/MM calculations including only the static charges of the water solvent, neglecting explicit polarization effects, were also state-averaged over 12 states. This allows us to estimate the effect of electronic polarization by comparison of QM/MM and QM/MMpol calculations, where the same sets of water partial charges are used. We note, however, that this set of charges describe the charge distribution of water in gas phase, as the increased polarization in condensed phase is accounted for through the water polarizability. We have also performed test CASSCF calculations on several snapshots using instead water charges from the TIP3P model, ${ }^{47}$ which include condensed-phase polarization effects implicitly. Our results indicate that QM/MM TIP3P calculations are able to recover about $60 \%$ of the polarization shifts in transition energies observed when comparing $\mathrm{QM} / \mathrm{MM}$ and $\mathrm{QM} / \mathrm{MMpol}$ calculations based on the same water charge model, which illustrates the importance of explicitly accounting for solvent polarization effects. The set of PCM polarization charges and the QM/MMpol and $\mathrm{QM} / \mathrm{MM}$ sets of charges and induced dipoles were then given as input in subsequent CASSCF, CASPT2 and MS-CASPT2 calculations performed with the Molcas 7.6 
package $^{50}$ using the ESPF module. An IPEA parameter of 0.25 , which corrects for systematic errors of the original CASPT2 formulation, ${ }^{51}$ was used in all CASPT2 calculations. This value is the current default and has been optimized for the spectroscopic parameters of benzene and a set of diatomic molecules. We also used an imaginary level shift ${ }^{52}$ of 0.1 in all CASPT2 calculations. The MS-CASPT2 oscillator strengths were calculated with the transition dipole moments from the perturbationally modified complete active space configuration interaction (PM-CASCI) wave function, ${ }^{26}$ which is obtained applying the unitary transformation of the multi-state procedure to the CASSCF wave function.

QM/MMpol calculations were performed using the polarizable MM parameters (charges and isotropic polarizabilities) for water developed in Ref.[ $\left[{ }^{15}\right]$. In all QM/MMpol calculations, only water molecules located at distances closer than $20 \AA$ to any QM atom were included as MM sites.

The dipole moments adopted by the water molecules in response to the SA density, examined for the 101 snapshots, fluctuated around an average of 3.01 Debye (standard deviation of 0.38 Debye), and no particularly large dipoles that could suggest numerical problems were found. Slightly larger dipole moments where found for water molecules interacting with the cytosine NH groups ( $\sim 3.17$ Debye) and the $\mathrm{N}(\sim 3.10$ Debye) and O group ( 3.14 Debye), however, with a comparable standard deviation ( 0.31 Debye) to that found for the other water molecules. For selected snapshots, true state-specific calculations were performed using state-optimized orbitals, where the solvent polarization adapted to the density of the state of interest. In this latter case, however, no particular trends were found when comparing the dipoles of hydrogenbonded water molecules induced by the SA, the ground state, or the first excited state densities.

\section{Results and discussion}

In Table 1 we report the MS-CASPT2 transition energies and oscillator strengths calculated in vacuum and in water with four different approaches: PCM, QM/MM and QM/MMpol with and without the state-specific correction. The QM/MM and QM/MMpol results are averages over the 101 selected snapshots. A detailed comparison with the experimentally assigned bands I-IV is provided in Table 2, and a graphical summary of the solvent shifts on the different states in Figure 2. For 
completeness, the CASSCF and CASPT2 data are provided in the Supporting Information (Tables SI1 and SI2). In vacuum, the first eleven excited states involve six $\pi \rightarrow \pi^{*}$ and five $n \rightarrow \pi^{*}$ transitions, whereas in the presence of the PCM or QM/MM environment these correspond to seven $\pi \rightarrow \pi^{*}$ and four $n \rightarrow \pi^{*}$ excited states. We center our analysis on the four lowest $\pi \rightarrow \pi^{*}$ states, for which the experimental data are available, and the four $n \rightarrow \pi^{*}$ states, whose energy falls in the same range. The dominant orbital transitions for these states are included in Table 1, and the orbitals are displayed in Figure 3.

The $n \rightarrow \pi^{*}$ states in vacuum appear between 5.3 and $7.1 \mathrm{eV}$, and their oscillator strength $f$ is smaller than 0.01. At the MS-CASPT2 level, the lowest states $n \rightarrow \pi^{*}$ can be described as excitations from the delocalized $n$ orbitals (linear combinations of the oxygen- and nitrogen-centered lone pairs, see Figure 3). In solution, the three lowest states are shifted up in energy by $0.9-1.0 \mathrm{eV}$, and $f$ increases to $0.02-0.06$. This is in line with what is expected for $n \rightarrow \pi^{*}$ states. The increase in the excitation energy comes from stabilization of the electron lone pairs by the solvent. In turn, the increase of the oscillator strength is due to mixing of the $\pi \rightarrow \pi^{*}$ and $n \rightarrow \pi^{*}$ states favored by the environment, which breaks the symmetry and allows for the mixing. Previous studies on cytosine monohydrate with PCM give a shift of 0.6 and $0.8 \mathrm{eV}$ for the two lowest $n \rightarrow \pi^{*}$ states,${ }^{39}$ and similar results $(0.56$ and $0.83 \mathrm{eV})$ are obtained with QM/MM. ${ }^{38}$ As could be expected, the shifts calculated with QM/MMpol are higher, approximately $1.0 \mathrm{eV}$ for both states.

The $\pi \rightarrow \pi^{*}$ states follow different trends. The two lowest states, of $\pi_{H} \rightarrow \pi_{L}^{*}$ and $\pi_{H-1} \rightarrow \pi_{L}^{*}$ character, are shifted up by approximately $0.1 \mathrm{eV}$ in the QM/MMpol approximation, and by smaller amounts with PCM and QM/MM. These small blue shifts are due to the fact that the excited states have smaller dipole moments than the ground state (4.1 and 5.4 Debye against 6.2 Debye for the ground state, see Table SI3 in the Supporting Information), which leads to an increase of the excitation energy in the polar environment. In contrast, the $\pi_{H} \rightarrow \pi_{L+1}^{*}$ and $\pi_{H-1} \rightarrow \pi_{L+1}^{*}$ states, where the dipole moments are similar in magnitude to the ground state (6.1 and 6.4 Debye), are red shifted by 0.6 and $0.2 \mathrm{eV}$, respectively. The different shifts experienced, in this case, probably arise because the density rearrangement upon excitation to the $\pi_{H} \rightarrow \pi_{L+1}^{*}$ state interacts more favorably with the water configuration surrounding cytosine compared to 
that occurring in the $\pi_{H-1} \rightarrow \pi_{L+1}^{*}$ state. Together with the excitation energy shifts, changes in the oscillator strengths of the $\pi \rightarrow \pi^{*}$ states are also observed upon solvation. The order of intensity of the experimental bands is IV $>$ III $>$ I $>$ II. The vacuum calculations give a different order in terms of oscillator strengths, where band III of the spectrum $\left(\pi_{H} \rightarrow \pi_{L+1}^{*}\right.$ state) has the strongest absorption and band I ( $\pi_{H} \rightarrow \pi_{L}^{*}$ state) the weakest one. This order is maintained with the PCM and QM/MM approaches, and the experimental order of intensities is only reproduced with the QM/MMpol approach. Although a comparison with experimental intensities is not straightforward, our results indicate a better accord with experiments in the QM/MMpol results compared to PCM and QM/MM calculations.

Examination of the MS-CASPT2 results with different solvation methods shows that in most cases the solvent shifts follow the same trend (see Figure 2c). The only exception is the $\pi_{H-1} \rightarrow \pi_{L}^{*}$ state, which is slightly red-shifted with QM/MM and blueshifted with PCM and QM/MMpol. This is probably due to the fact that the absolute value of the shift is small. For the remaining states, all methods predict the same qualitative trend. In most cases the shifts also increase in the order PCM $<\mathrm{QM} / \mathrm{MM}<$ $\mathrm{QM} / \mathrm{MMpol}<\mathrm{QM} / \mathrm{MMpol}+\mathrm{SScorr}$. The trend between PCM and QM/MM shifts probably arises due to the inclusion of specific solute-solvent interactions, in particular hydrogen bonds, in the latter. On the other hand, explicit inclusion of polarization effects enhances the solute-solvent interactions and as a result, the solvent shifts in QM/MMpol are larger compared to QM/MM. Interestingly, also the state-specific correction further increases these shifts. Overall, it is quite remarkable that the PCM shifts shown in Figure 2 are close to the QM/MM estimates, which illustrates the ability of the PCM model to describe homogeneous solvents. In heterogeneous environment like those commonly found in biological systems, however, the QM/MM models are expected to significantly improve the PCM description, given their ability to account for the structure of the local environment. ${ }^{20}$

Turning to the other electronic structure methods (see Figure 2), in most cases the CASSCF shifts follow the same trend observed for MS-CASPT2, but in several cases they are substantially overestimated. For instance, the QM/MMpol shifts including the state specific correction for the three lowest $n \rightarrow \pi^{*}$ states are $1.2-1.7 \mathrm{eV}$ at the CASSCF level and $0.9-1.0 \mathrm{eV}$ at the MS-CASPT2 level. The overestimated 
CASSCF shifts can be understood in terms of the electronic density of the solute. The large shifts of the $n \rightarrow \pi^{*}$ states are caused by large changes in the density upon excitation (see the changes in the dipole moments in Table SI3). At the state-averaged CASSCF level, these changes are described for the active space orbitals. At the CASPT2 level, dynamic correlation allows for a polarization of the $\sigma$ electron density that compensates the polarization of the active space orbitals, and the differences of the total density with respect to the ground state density are reduced. This results in a smaller solvatochromic shift. In fact, the dipole moment differences between the $n \rightarrow \pi^{*}$ states and the ground state reflects this behavior. They decrease from 4.0 - 4.6 Debye at the CASSCF level to $3.3-4.1$ Debye with CASPT2 (see Table SI3). The $\pi_{H} \rightarrow \pi_{L}^{*}$ state behaves similarly, since its blue-shift is overestimated at the CASSCF level $(0.5 \mathrm{eV})$ with respect to MS-CASPT2 $(0.1 \mathrm{eV})$. The important point here is that the CASPT2 treatment corrects the overestimated CASSCF shifts: the solvent polarization response is overestimated by CASSCF, but the use of the CASPT2 density for the energy calculation corrects this effect.

The agreement between the MS-CASPT2 vertical absorption energies calculated with our QM/MMpol approach and the experimental bands is satisfactory, with differences between 0.17 and $0.59 \mathrm{eV}$. These errors are somewhat larger than expected, and there are possible sources of error. First, our calculations do not consider the internal degrees of freedom of cytosine. Instead, the energies are calculated as vertical excitations on the ground state minimum geometry with different solvent conformations. Recent studies indicate that the calculated vertical excitation energies tend to overestimate the frequency of the absorption band maximum between 0.1 and $0.3 \mathrm{eV}$ due to the limitations of the Franck-Condon approximation, and this explains part of the deviation from experiment found in our results. In addition, fluctuations of cytosine internal geometry can lead to mixing among $n \rightarrow \pi^{*}$ and $\pi \rightarrow \pi^{*}$ states, and such mixing can alter the positions of the absorption maxima. Our calculations also do not include other, minor tautomers. In this case, however, theoretical studies based on continuum solvation models and free energy simulations suggest that the keto $\mathrm{N} 1 \mathrm{H}$ tautomer is preferred over other forms by more than $5 \mathrm{kcal} \mathrm{mol} .^{28}$ Finally, for some of the states dynamic correlation has a large contribution to the excitation energy, and in this case our approach of solving the polarization only at the CASSCF level may be insufficient to obtain the full solvent effect on the excitation. 
To compare the different solvation approaches, in Table 2 we provide the mean error for each solvation method, obtained as the difference between the calculated excitations and the experimental band maxima, averaged over the four bands. The mean errors indicate that, for the $\pi \rightarrow \pi^{*}$ states, the calculated excitations approach the experimental values as one goes from $\mathrm{PCM}$ to $\mathrm{QM} / \mathrm{MM}$ and $\mathrm{QM} / \mathrm{MMpol}$. Regarding the band intensities, only the QM/MMpol approach gives the right order of bands. However, the relative intensity is not recovered correctly. According to the analysis of Ref. $\left[{ }^{41}\right]$, the most intense band IV, is more than six times as intense as band II, which is the weakest one. In contrast, the calculated oscillator strength for band IV is only twice as large as that of band II. Such a large discrepancy is probably due to several causes. First, the contribution of dynamic correlation to the excitation energy of this state in vacuum is more than $1 \mathrm{eV}$, and in this case our approach may not account for the full polarization effect, as noted above. The $n_{1} \rightarrow \pi_{L}^{*}$ transition, which is calculated at 6.82 $\mathrm{eV}$ and has an oscillator strength of 0.06 , may also contribute to band IV, making it more intense than what is predicted by our calculations for the $\pi_{H} \rightarrow \pi_{L+1}^{*}$ transition.

Regarding the state-specific correction, at the MS-CASPT2 level it amounts in most cases to $15-20 \%$ of the overall QM/MMpol shift. The states with the largest correction are the three lowest $n \rightarrow \pi^{*}$ states and the $\pi_{H} \rightarrow \pi_{L+1}^{*}$ state, which also have the largest shifts. In these cases, the correction follows the same trend as the QM/MMpol shift, i.e. blue-shifted states are further shifted to the blue and red-shifted ones to the red. Focusing on the four lowest $\pi \rightarrow \pi^{*}$ states, the comparison of the corrected and uncorrected QM/MMpol excitations with the experimental bands gives virtually no improvement when the corrections are included (see the mean errors in Table 2). However, the comparison between the vertical excitations and the experimental band maxima has its limitations, as discussed above. To gain further insight into the benefits of the proposed correction scheme, we have compared it with the results of "fully" state-specific calculations using state-optimized orbitals. The excitations of $S_{1}$ have been calculated with the QM/MMpol scheme, using state-optimized orbitals, for five snapshots (see Table 3). These excitations, labeled $\Delta E_{S O}$, are obtained from two independent calculations for $S_{1}$ and $S_{0}$. The values of $\Delta E_{S O}$ are compared with the stateaveraged QM/MMpol excitations without and with the state-specific correction $\left(\Delta E_{S A}\right.$ and $\Delta E_{S S}$, respectively). 
The QM/MMpol $\Delta E_{S O}$ values are more blue-shifted with respect to the vacuum excitation compared to $\Delta E_{S A}$ state-averaged ones by $\sim 0.13 \mathrm{eV}$. This difference is inherent to the two schemes. On one hand, the SA-scheme only gives a partial account of the solvent effect because the density of the states and the polarization of the solvent are not fully consistent. Beyond solvent effects, however, the state-averaging procedure has its own limitations, as illustrated by the similar deviation of $\sim 0.14 \mathrm{eV}$ found between $\Delta E_{S O}$ and $\Delta E_{S A}$ results in analogous calculations performed in vacuum. This suggests that the major source of error is the state-averaging procedure itself, rather than the solvation model and the state-specific correction. Indeed, the state-specific correction reduces the deviation in $\mathrm{QM} / \mathrm{MMpol}$ results from $\sim 0.13 \mathrm{eV}$ to $\sim 0.11 \mathrm{eV}$, a value smaller than that found in gas phase. The main point is that in all snapshots the state-specific correction brings the shifts closer to the state-optimized values, and the corrected values are improved compared to the uncorrected ones. It would be desirable to carry out a similar test for the remaining excited states, but this was not possible because state-optimized calculations for higher states did not converge. However, the fact that the proposed corrections follow the correct trend for $S_{1}$ suggests that the corrections for the other states, which are larger in magnitude, also bring the calculated shifts closer to the more accurate, state-optimized results.

\section{Conclusions}

We have implemented a polarizable QM/MM model at the CASSCF level of theory and applied it to the calculation of the vertical absorption spectrum of the canonical tautomer of cytosine in water. In our approach, the mutual polarization of the solute and the solvent is solved self-consistently at the CASSCF level, and the resulting set of charges and dipoles is used to calculate the vertical excitation energies at the CASPT2 and MS-CASPT2 levels of theory. Our scheme allows treating multiple excited states by converging the solvent polarization with respect to the state-averaged density of the solute. We also propose a state-specific correction where the solvent polarization is recomputed with the density of each state and the CASSCF energies recalculated with the corresponding polarization. Comparison of the QM/MMpol excitation energy of $S_{1}$, computed with state-averaged orbitals and state-specific correction, with that computed with state-optimized orbitals shows that the state- 
specific correction brings the excitation energies closer to the values obtained with state-optimized orbitals.

The seven lowest $\pi \rightarrow \pi^{*}$ states and the four lowest $n \rightarrow \pi^{*}$ states of cytosine have been considered. At the MS-CASPT2 level, the three lowest $n \rightarrow \pi^{*}$ states are shifted up in energy by approximately $1 \mathrm{eV}$ with the QM/MMpol. The blue shifts of these states increase in magnitude going from $\mathrm{PCM}$ to $\mathrm{QM} / \mathrm{MM}$ and $\mathrm{QM} / \mathrm{MMpol}$, which is in agreement with the accepted view that specific solute-solvent interactions, in the form of hydrogen bonds, are important for the excitations of these states. For the $\pi \rightarrow \pi^{*}$ states, the comparison of the calculated four lowest $\pi \rightarrow \pi^{*}$ states with the band maxima from the experimental spectrum shows that the best agreement between theory and experiment, both regarding the energy of the bands and their intensity, is obtained with the QM/MMpol approach. Overall, these results show the validity of our approach for the study of excited states in solution. Some limits are however evident: some of the calculated $\pi \rightarrow \pi^{*}$ excitations show substantial deviations from the experimental bands, of up to $0.54 \mathrm{eV}$. This may be due, in part, to the fact that the polarization of the solvent is calculated at the CASSCF level and not at the CASPT2 or MS-CASPT2 levels, and this may be insufficient in the cases where the contribution of dynamic correlation energy is important, such as band IV of the spectrum. Beyond solvent effects, however, the large magnitude of correlation energy found in this band suggests that the perturbative treatment of dynamical correlation effects in CASPT2 and MS-CASPT2 methods could also explain the deviations observed.

Our results are relevant for the photophysics of the DNA nucleobases. For cytosine and cytidine monophosphate it has been suggested that a dark $n \rightarrow \pi^{*}$ state is responsible for a decay component with a lifetime of 12 - 34 ps in water solution ${ }^{53}$ after excitation with $267 \mathrm{~nm}$ light (approximately $4.64 \mathrm{eV}$ ). The vertical excitations calculated here for these states, which are higher than $6 \mathrm{eV}$ and are in line with previous results with other methods, ${ }^{38-39}$ speak against this possibility. However, it cannot be excluded that the $n \rightarrow \pi^{*}$ state may be lower in energy in other regions of the potential energy surface and may be populated after internal conversion from $S_{1}$. This is a question that cannot be settled here, since it needs a study of the potential energy surface that requires having the gradients for the QM/MMpol method. To this end we plan to extend the gradients recently implemented at the linear response level $^{54}$ to 
MCSCF. This implementation will also allow the determination of conical intersections in solution.

Beyond calculations in solvents, our scheme will also be useful for the study of excited states of biological systems, where environmental effects and specific interactions between the chromophore and the environment are important. The method will also be useful for the treatment of multichromophoric systems in solution, where charge transfer and exciton states are relevant. For the latter, inclusion of explicit MM polarization is particularly important in order to recover the solvent screening effects in electronic couplings among excited states that lead to exciton delocalization. ${ }^{20}$ This will be the subject of further future work.

\section{Acknowledgements}

C.C. and L.B. acknowledge support from the Ministerio de Economía y Competitividad (MINECO) of Spain (C.C.: grants CTQ2012-36195 and RYC2011-08918; L.B.: grants CTQ2011- 26573 and UNGI10-4E-801 from FEDER (European Fund for Regional Development)), the Agència de Gestió d'Ajuts Universitaris i de Recerca from Generalitat de Catalunya (GENCAT) (C.C.: SGR2014-1189; L.B.: 2014SGR1202), and the Direcció General de la Recerca (GENCAT) for the Xarxa de Referència en Química Teòrica i Computacional de Catalunya. C.C. also acknowledges computational resources provided by the Consorci de Serveis Universitaris de Catalunya. Q. L. acknowledges the National Natural Science Foundation of China (21303007) and the Excellent Young Scholars Research Fund of Beijing Institute of Technology (2013YR1917).

Supporting Information. Transition energies and oscillator strengths obtained in vacuum and with different solvation approaches at the CASSCF and CASPT2 levels of theory (Tables SI1 and SI2, respectively), dipole moments obtained in vacuum at the CASSCF and CASPT2 levels of theory (Table SI3). Cartesian coordinates of cytosine. This material is available free of charge via the Internet at http://pubs.acs.org.

\section{References}


1. Rosspeintner, A.; Lang, B.; Vauthey, E. Annu. Rev. Phys. Chem. 2013, 64, 247-271.

2. Mennucci, B. Phys. Chem. Chem. Phys. 2013, 15, 6583-6594.

3. Gonzalez, L.; Escudero, D.; Serrano-Andres, L. ChemPhysChem 2012, 13, 28-51.

4. Severo Pereira Gomes, A.; Jacob, C. R. Ann. Rep. Prog. Chem. Sect. C 2012, 108, $222-$

277.

5. $\quad$ Kohler, B. J. Phys. Chem. Lett. 2010, 1, 2047-2053.

6. Markovitsi, D.; Gustavsson, T.; Vaya, I. J. Phys. Chem. Lett. 2010, 1, 3271-3276.

7. Ha, T.; Tinnefeld, P. Annu. Rev. Phys. Chem. 2012, 63, 595-617.

8. Thompson, M. A.; Schenter, G. K. J. Phys. Chem. 1995, 99, 6374-6386.

9. Jensen, L.; van Duijnen, P. T.; Snijders, J. G. J. Chem. Phys. 2003, 119, 3800-3809.

10. Kongsted, J.; Osted, A.; Mikkelsen, K. V.; Christiansen, O. J. Chem. Phys. 2003, 118, 1620-1633.

11. Ohrn, A.; Karlstrom, G. Mol. Phys. 2006, 104, 3087-3099.

12. Nielsen, C. B.; Christiansen, O.; Mikkelsen, K. V.; Kongsted, J. J. Chem. Phys. 2007, $126,154112$.

13. Lin, Y. L.; Gao, J. L. J. Chem. Theory Comput. 2007, 3, 1484-1493.

14. Yoo, S.; Zahariev, F.; Sok, S.; Gordon, M. S. J. Chem. Phys. 2008, 129, 144112.

15. Curutchet, C.; Munoz-Losa, A.; Monti, S.; Kongsted, J.; Scholes, G. D.; Mennucci, B. J. Chem. Theory Comput. 2009, 5, 1838-1848.

16. Soderhjelm, P.; Husberg, C.; Strambi, A.; Olivucci, M.; Ryde, U. J. Chem. Theory Comput. 2009, 5, 649-658.

17. Hedegard, E. D.; Jensen, H. J. A.; Kongsted, J. Int. J. Quantum Chem. 2014, 114, 11021107.

18. Thellamurege, N. M.; Si, D. J.; Cui, F. C.; Zhu, H. B.; Lai, R.; Li, H. J. Comput. Chem. 2013, 34, 2816-2833.

19. Warshel, A.; Levitt, M. J. Mol. Biol. 1976, 103, 227-249.

20. $\quad$ Curutchet, C.; Kongsted, J.; Muñoz-Losa, A.; Hossein-Nejad, H.; Scholes, G. D.; Mennucci, B. J. Am. Chem. Soc. 2011, 133, 3078-3084.

21. Viani, L.; Curutchet, C.; Mennucci, B. J. Phys. Chem. Lett. 2013, 4, 372-377.

22. Steindal, A. H.; Ruud, K.; Frediani, L.; Aidas, K.; Kongsted, J. J. Phys. Chem. B 2011, 115, 3027-3037.

23. Thellamurege, N. M.; Cui, F.; Li, H. J. Chem. Phys. 2013, 139.

24. Roos, B. O.; Taylor, P. R.; Siegbahn, P. E. M. Chem. Phys. 1980, 48, 157-173.

25. Andersson, K.; Malmqvist, P. A.; Roos, B. O.; Sadlej, A. J.; Wolinski, K. J. Phys. Chem. 1990, 94, 5483-5488.

26. Finley, J.; Malmqvist, P. A.; Roos, B. O.; Serrano-Andres, L. Chem. Phys. Lett. 1998, 288, 299-306.

27. Hedegard, E. D.; List, N. H.; Jensen, H. J. A.; Kongsted, J. J. Chem. Phys. 2013, 139.

28. Colominas, C.; Luque, F. J.; Orozco, M. J. Am. Chem. Soc. 1996, 118, 6811-6821.

29. Crespo-Hernandez, C. E.; Cohen, B.; Hare, P. M.; Kohler, B. Chem. Rev. 2004, 104, 1977-2019.

30. Abouaf, R.; Pommier, J.; Dunet, H.; Quan, P.; Nam, P. C.; Nguyen, M. T. J. Chem. Phys. 2004, 121, 11668-11674.

31. Szalay, P. G.; Watson, T.; Perera, A.; Lotrich, V. F.; Bartlett, R. J. J. Phys. Chem. A 2012, 116, 6702-6710.

32. Schreiber, M.; Silva, M. R.; Sauer, S. P. A.; Thiel, W. J. Chem. Phys. 2008, 128.

33. Blancafort, L. Photochem. Photobiol. 2007, 83, 603-610.

34. Kistler, K. A.; Matsika, S. J. Phys. Chem. A 2007, 111, 2650-2661.

35. Tomic, K.; Tatchen, J.; Marian, C. M. J. Phys. Chem. A 2005, 109, 8410-8418.

36. Avila Ferrer, F. J.; Santoro, F.; Improta, R. Comp. Theor. Chem. 2014, 1040, 186-194.

37. Aquino, A. J. A.; Nachtigallova, D.; Hobza, P.; Truhlar, D. G.; Hattig, C.; Lischka, H. J. Comput. Chem. 2011, 32, 1217-1227.

38. Kistler, K. A.; Matsika, S. J. Phys. Chem. A 2009, 113, 12396-12403.

39. Blancafort, L.; Migani, A. J. Photochem. Photobiol. A-Chem. 2007, 190, 283-289. 
40. Domingo, A.; Rodriguez-Fortea, A.; de Graaf, C. J. Chem. Theory Comput. 2012, 8 , 235-244.

41. Zaloudek, F.; Novros, J. S.; Clark, L. B. J. Am. Chem. Soc. 1985, 107, 7344-7351.

42. Mennucci, B. WIREs Comput. Mol. Sci. 2012, 2, 386-404.

43. Avila Ferrer, F. J.; Cerezo, J.; Stendardo, E.; Improta, R.; Santoro, F. J. Chem. Theory Comput. 2013, 9, 2072-2082.

44. $\quad$ Curutchet, C.; Novoderezhkin, V. I.; Kongsted, J.; Muñoz-Losa, A.; van Grondelle, R.; Scholes, G. D.; Mennucci, B. J. Phys. Chem. B 2013, 117, 4263-4273.

45. Cossi, M.; Barone, V.; Robb, M. A. J. Chem. Phys. 1999, 111, 5295-5302.

46. Case, D. A.; Darden, T. A.; Cheatham, I., T.E.; Simmerling, C. L.; Wang, J.; Duke, R. E.; Luo, R.; Merz, K. M.; Pearlman, D. A.; Crowley, M.; Walker, R. C.; Zhang, W.; Wang, B.; Hayik, S.; Roitberg, A.; Seabra, G.; Wong, K. F.; Paesani, F.; Wu, X.; Brozell, S.; Tsui, V.; Gohlke, H.; Yang, L.; Tan, C.; Mongan, J.; Hornak, V.; Cui, G.; Beroza, P.; Mathews, D. H.; Schafmeister, C.; Ross, W. S.; Kollman, P. A. AMBER 9, University of California: San Francisco, 2006.

47. Jorgensen, W. L.; Chandrasekhar, J.; Madura, J. D.; Impey, R. W.; Klein, M. L. J. Chem. Phys. 1983, 79, 926-935.

48. Wang, J. M.; Cieplak, P.; Kollman, P. A. J. Comput. Chem. 2000, 21, 1049-1074.

49. Frisch, M. J.; Trucks, G. W.; Schlegel, H. B.; Scuseria, G. E.; Robb, M. A.; Cheeseman, J. R.; Scalmani, G.; Barone, V.; Mennucci, B.; Petersson, G. A.; Nakatsuji, H.; Caricato, M.; Li, X.; Hratchian, H. P.; Izmaylov, A. F.; Bloino, J.; Zheng, G.; Sonnenberg, J. L.; Hada, M.; Ehara, M.; Toyota, K.; Fukuda, R.; Hasegawa, J.; Ishida, M.; Nakajima, T.; Honda, Y.; Kitao, O.; Nakai, H.; Vreven, T.; Montgomery, J., J. A.; Peralta, J. E.; Ogliaro, F.; Bearpark, M.; Heyd, J. J.; Brothers, E.; Kudin, K. N.; Staroverov, V. N.; Kobayashi, R.; Normand, J.; Raghavachari, K.; Rendell, A.; Burant, J. C.; Iyengar, S. S.; Tomasi, J.; Cossi, M.; Rega, N.; Millam, N. J.; Klene, M.; Knox, J. E.; Cross, J. B.; Bakken, V.; Adamo, C.; Jaramillo, J.; Gomperts, R.; Stratmann, R. E.; Yazyev, O.; Austin, A. J.; Cammi, R.; Pomelli, C.; Ochterski, J. W.; Martin, R. L.; Morokuma, K.; Zakrzewski, V. G.; Voth, G. A.; Salvador, P.; Dannenberg, J. J.; Dapprich, S.; Daniels, A. D.; Farkas, Ö.; Foresman, J. B.; Ortiz, J. V.; Cioslowski, J.; Fox, D. J. Gaussian 09, Revision A.2, Gaussian, Inc.: Wallingford CT, 2009.

50. $\quad$ Aquilante, F.; De Vico, L.; Ferre, N.; Ghigo, G.; Malmqvist, P.-A.; Neogrady, P.; Pedersen, T. B.; Pitonak, M.; Reiher, M.; Roos, B. O.; Serrano-Andres, L.; Urban, M.; Veryazov, V.; Lindh, R. J. Comput. Chem. 2010, 31, 224-247.

51. Ghigo, G.; Roos, B. O.; Malmqvist, P. A. Chem. Phys. Lett. 2004, 396, 142-149.

52. Forsberg, N.; Malmqvist, P. A. Chem. Phys. Lett. 1997, 274, 196-204.

53. Hare, P. M.; Crespo-Hernandez, C. E.; Kohler, B. Proc. Natl. Acad. Sci. U. S. A. 2007, 104, 435-440.

54. $\quad$ Caprasecca, S.; Jurinovich, S.; Viani, L.; Curutchet, C.; Mennucci, B. J. Chem. Theory Comput. 2014, 10, 1588-1598. 
Table 1. MS-CASPT2 $(14,10) / \mathrm{cc}-\mathrm{pVTZ}$ state-averaged transition energies $\left(\Delta E_{S A}\right)$ and oscillator strengths $(f)$ computed for the four lowest $n \rightarrow \pi^{*}$ and seven lowest $\pi \rightarrow \pi^{*}$ transtions of cytosine in vacuum and in water as described by PCM, QM/MM and polarizable QM/MM calculations. For the latter, the state-specific corrections $\left(\Delta E_{\text {corr }}\right)$ as well as the state-specific corrected transition energies $\left(\Delta E_{S S}=\Delta E_{S A}+\Delta E_{\text {corr }}\right)$ are also shown.

\begin{tabular}{|c|c|c|c|c|c|c|c|}
\hline & & Vacuum & PCM & $\mathrm{QM} / \mathrm{MM}$ & \multicolumn{3}{|c|}{ QM/MMpol } \\
\hline Character & State & $\begin{array}{c}\Delta E_{S A}{ }^{\mathrm{a}} \\
f\end{array}$ & $\begin{array}{l}\Delta E_{S A}{ }^{\mathrm{a}} \\
f\end{array}$ & $\begin{array}{c}\Delta E_{S A}^{\mathrm{a}, \mathrm{b}} \\
f\end{array}$ & $\begin{array}{l}\Delta E_{S A}^{\mathrm{a}, \mathrm{b}} \\
f\end{array}$ & $\Delta E_{c o r r}^{\mathrm{a}, \mathrm{b}, \mathrm{c}}$ & $\Delta E_{S S}=\Delta E_{S A}+\Delta E_{c o r r}$ \\
\hline$n_{2} \rightarrow \pi_{L}^{*}$ & 1 & $\begin{array}{c}5.275 \\
0.00\end{array}$ & $\begin{array}{c}5.785 \\
0.00\end{array}$ & $\begin{array}{c}5.880 \\
0.01\end{array}$ & $\begin{array}{l}6.152 \\
0.02\end{array}$ & 0.124 & 6.276 \\
\hline$n_{1} \rightarrow \pi_{L}^{*}$ & 2 & $\begin{array}{c}5.795 \\
0.00\end{array}$ & $\begin{array}{c}6.497 \\
0.00\end{array}$ & $\begin{array}{c}6.573 \\
0.01\end{array}$ & $\begin{array}{c}6.684 \\
0.06\end{array}$ & 0.137 & 6.821 \\
\hline$n_{2} \rightarrow \pi_{L+1}^{*}$ & 3 & $\begin{array}{c}6.322 \\
0.00\end{array}$ & $\begin{array}{c}6.712 \\
0.00\end{array}$ & $\begin{array}{c}6.821 \\
0.04\end{array}$ & $\begin{array}{c}7.100 \\
0.02\end{array}$ & 0.117 & 7.217 \\
\hline$n_{1} \rightarrow \pi_{L+1}^{*}$ & 4 & $\begin{array}{c}7.141 \\
0.00 \\
\end{array}$ & $\begin{array}{c}7.141 \\
0.00 \\
\end{array}$ & $\begin{array}{c}7.199 \\
0.01 \\
\end{array}$ & $\begin{array}{c}7.376 \\
0.01 \\
\end{array}$ & 0.052 & 7.428 \\
\hline$\pi_{H} \rightarrow \pi_{L}^{*}$ & 1 & $\begin{array}{l}4.781 \\
0.14\end{array}$ & $\begin{array}{c}4.856 \\
0.18\end{array}$ & $\begin{array}{c}4.855 \\
0.22\end{array}$ & $\begin{array}{c}4.857 \\
0.28\end{array}$ & 0.013 & 4.870 \\
\hline$\pi_{H-1} \rightarrow \pi_{L}^{*}$ & 2 & $\begin{array}{c}5.614 \\
0.37\end{array}$ & $\begin{array}{c}5.621 \\
0.34\end{array}$ & $\begin{array}{c}5.576 \\
0.27\end{array}$ & $\begin{array}{c}5.667 \\
0.24\end{array}$ & 0.041 & 5.708 \\
\hline$\pi_{H} \rightarrow \pi_{L+1}^{*}$ & 3 & $\begin{array}{c}6.504 \\
0.64\end{array}$ & $\begin{array}{c}6.287 \\
0.56\end{array}$ & $\begin{array}{c}6.203 \\
0.56\end{array}$ & $\begin{array}{c}6.016 \\
0.41\end{array}$ & -0.127 & 5.889 \\
\hline$\pi_{H-1} \rightarrow \pi_{L+1}^{*}$ & 4 & $\begin{array}{c}7.078 \\
0.35\end{array}$ & $\begin{array}{c}6.954 \\
0.50\end{array}$ & $\begin{array}{c}6.893 \\
0.45\end{array}$ & $\begin{array}{c}6.830 \\
0.50\end{array}$ & 0.052 & 6.882 \\
\hline$\pi \rightarrow \pi^{*}$ & 5 & $\begin{array}{c}8.635 \\
0.13\end{array}$ & $\begin{array}{c}8.301 \\
0.22\end{array}$ & $\begin{array}{c}8.296 \\
0.21\end{array}$ & $\begin{array}{c}8.126 \\
0.21\end{array}$ & -0.025 & 8.101 \\
\hline$\pi \rightarrow \pi^{*}$ & 6 & $\begin{array}{c}9.317 \\
0.02\end{array}$ & $\begin{array}{c}9.033 \\
0.01\end{array}$ & $\begin{array}{c}8.980 \\
0.01\end{array}$ & $\begin{array}{c}8.949 \\
0.04\end{array}$ & 0.025 & 8.974 \\
\hline$\pi \rightarrow \pi^{*}$ & 7 & $--^{d}$ & 9.182 & 9.147 & 9.107 & 0.046 & 9.153 \\
\hline
\end{tabular}


${ }^{\mathrm{a}}$ Transition energies in $\mathrm{eV} .{ }^{\mathrm{b}}$ Excitations averaged over 101 snapshots. ${ }^{\mathrm{c} C a l c u l a t e d}$ at the CASSCF level. ${ }^{\mathrm{d}}$ In vacuum the 11 excited states correspond to $6 \pi \rightarrow \pi^{*}$ and $5 n \rightarrow \pi^{*}$ transitions. 
Table 2. Comparison between experimental maxima of the absorption bands of cytosine in water with predicted MS-CASPT2(14,10)/cc-pVTZ state-averaged transition energies $\left(\Delta E_{S A}\right)$ and oscillator strengths $(f)$ computed from PCM, QM/MM and polarizable QM/MM calculations. For the latter, state-specific corrected transition energies $\left(\Delta E_{S S}=\Delta E_{S A}+\Delta E_{c o r r}\right)$ are also shown.

\begin{tabular}{|c|c|c|c|c|c|c|c|c|c|c|c|}
\hline & \multicolumn{3}{|c|}{ Experiment $^{\mathrm{a}}$} & \multicolumn{2}{|c|}{ PCM } & \multicolumn{2}{|c|}{ QM/MM } & \multicolumn{4}{|c|}{ QM/MMpol } \\
\hline Band & $\lambda_{\max }^{b}$ & $E_{\max }^{b}$ & $I_{\text {rel }}{ }^{c}$ & $\begin{array}{c}\Delta E_{S A}{ }^{b} \\
(f)\end{array}$ & Err & $\begin{array}{c}\Delta E_{S A}^{b, d} \\
(f)\end{array}$ & Err & $\begin{array}{c}\Delta E_{S A}^{b, d} \\
(f)\end{array}$ & Err & $\Delta E_{S S}^{b, d}$ & Err \\
\hline I & 264 & 4.70 & 1.9 & $\begin{array}{c}4.86 \\
(0.18)\end{array}$ & 0.16 & $\begin{array}{c}4.86 \\
(0.22)\end{array}$ & 0.16 & $\begin{array}{c}4.86 \\
(0.28)\end{array}$ & 0.16 & 4.87 & 0.17 \\
\hline II & 233 & 5.32 & 1.0 & $\begin{array}{c}5.62 \\
(0.34)\end{array}$ & 0.31 & $\begin{array}{c}5.58 \\
(0.27)\end{array}$ & 0.26 & $\begin{array}{c}5.67 \\
(0.24)\end{array}$ & 0.35 & 5.71 & 0.39 \\
\hline III & 214 & 5.80 & 2.4 & $\begin{array}{c}6.29 \\
(0.56)\end{array}$ & 0.48 & $\begin{array}{c}6.20 \\
(0.56)\end{array}$ & 0.40 & $\begin{array}{c}6.02 \\
(0.41)\end{array}$ & 0.22 & 5.89 & 0.09 \\
\hline IV & 195 & 6.34 & 6.6 & $\begin{array}{c}6.95 \\
(0.50)\end{array}$ & 0.61 & $\begin{array}{c}6.89 \\
(0.45)\end{array}$ & 0.55 & $\begin{array}{c}6.83 \\
(0.50)\end{array}$ & 0.49 & 6.88 & 0.54 \\
\hline Mean & & & & & 0.39 & & 0.34 & & 0.31 & & 0.30 \\
\hline
\end{tabular}

${ }^{\mathrm{a}} \operatorname{Ref}\left[{ }^{41}\right] .{ }^{\mathrm{b}}$ Wavelengths in $\mathrm{nm}$ and transition energies in $\mathrm{eV} .{ }^{\mathrm{c}}$ Relative intensity estimated assuming a Gaussian line shape for the bands of Ref $\left[{ }^{41}\right]$.

${ }^{\mathrm{d}}$ Excitations averaged over 101 snapshots. 
Table 3. CASSCF $(14,10) / c c-p V T Z$ excitation energies for $S_{1}$ calculated with the $\mathrm{QM} / \mathrm{MMpol}$ scheme using state-optimized energies $\left(\Delta E_{S O}\right)$ and state-averaged energies with and without the state-specific correction $\left(\Delta E_{S S}\right.$ and $\left.\Delta E_{S A}\right)$.

\begin{tabular}{ccccc}
\hline Snapshot & $\Delta E_{S O}{ }^{\mathrm{a}}$ & $\Delta E_{S A}^{\mathrm{b}}$ & $\Delta E_{\text {corr }}^{\mathrm{c}}$ & $\Delta E_{S S}=\Delta E_{S A}+\Delta E_{\text {Corr }}{ }^{\mathrm{d}}$ \\
\hline 1 & 5.602 & 5.476 & 0.022 & 5.498 \\
2 & 5.621 & 5.483 & 0.028 & 5.511 \\
3 & 5.679 & 5.543 & 0.018 & 5.561 \\
4 & 5.707 & 5.584 & 0.018 & 5.602 \\
5 & 5.610 & 5.498 & 0.02 & 5.518
\end{tabular}

${ }^{a}$ Excitation energy from two independent, state-specific calculations for $S_{1}$ and $S_{0}$. ${ }^{b}$ Excitation energy from a state-averaged calculation. ${ }^{\mathrm{c}}$ State-specific correction to the QM/MMpol transition energy (see Eqs. 8-10). ${ }^{\mathrm{d}} \mathrm{QM} / \mathrm{MMpol}$ excitation energy with state-specific correction. 
<smiles>Nc1cc[nH]c(=O)n1</smiles>

Figure 1. Chemical structure of the canonical cytosine keto $\mathrm{N} 1 \mathrm{H}$ tautomer in aqueous solution. 
a)

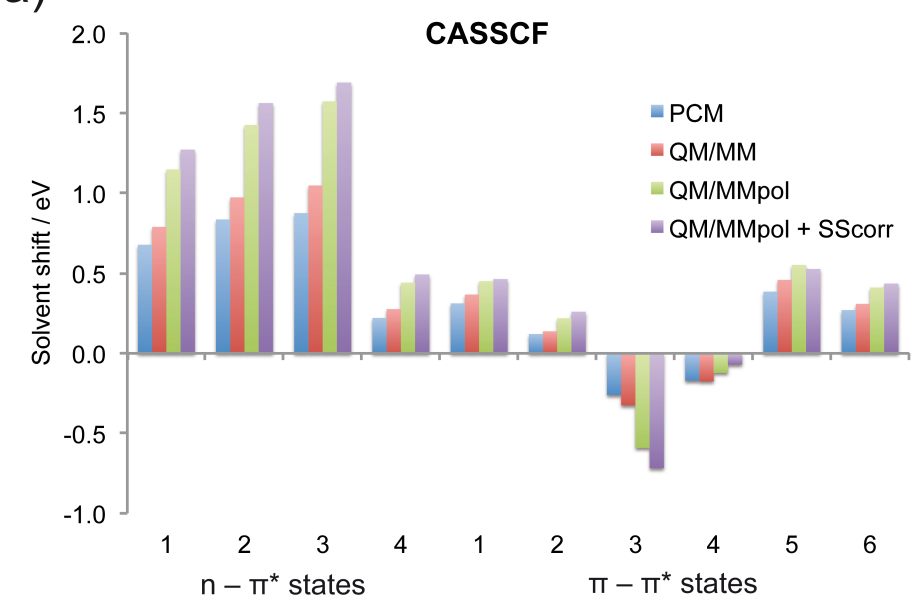

b)

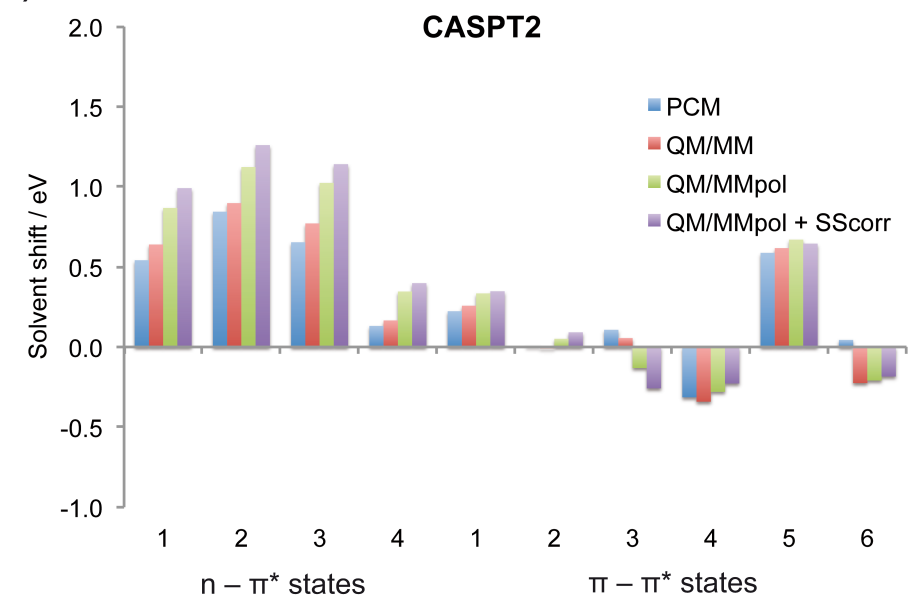

c)

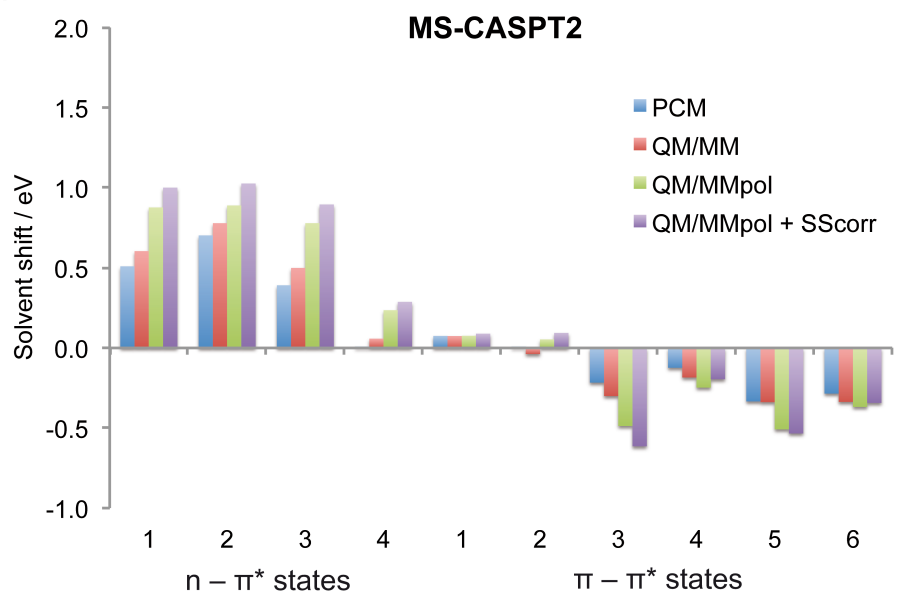

Figure 2. Solvent shifts experienced by the excited states of cytosine predicted by a) CASSCF, b) CASPT2 and c) MS-CASPT2 calculations based on the PCM and QM/MM models. 


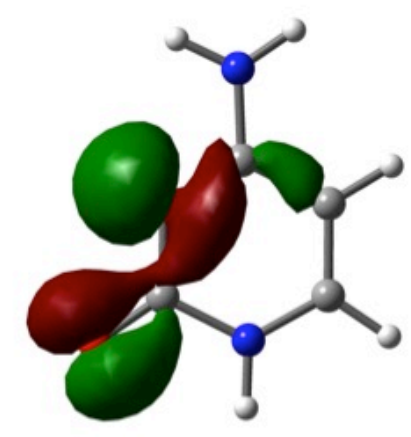

$n_{1}$

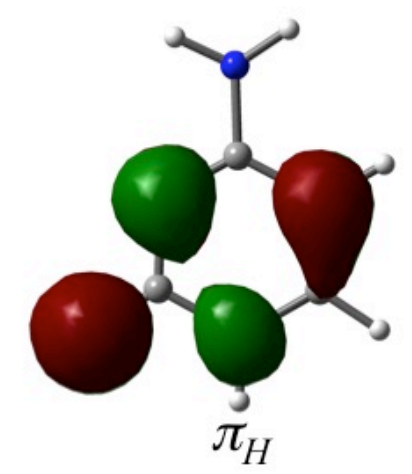

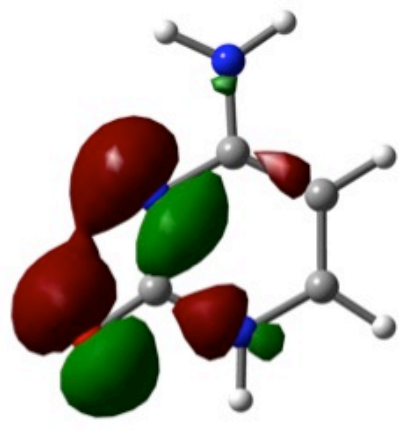

$n_{2}$

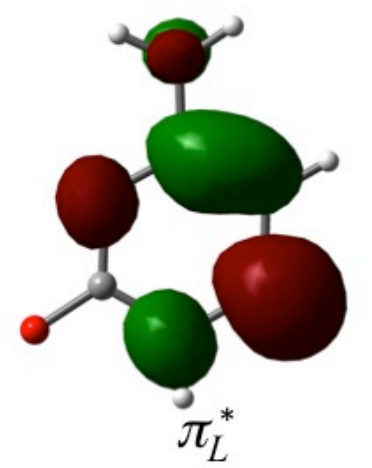

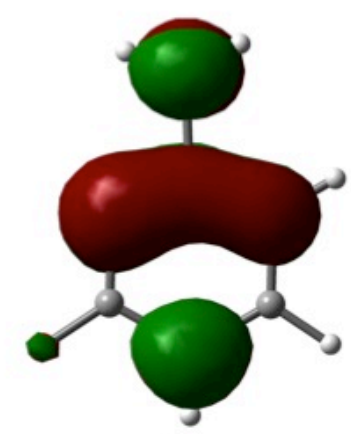

$\pi_{H-1}$

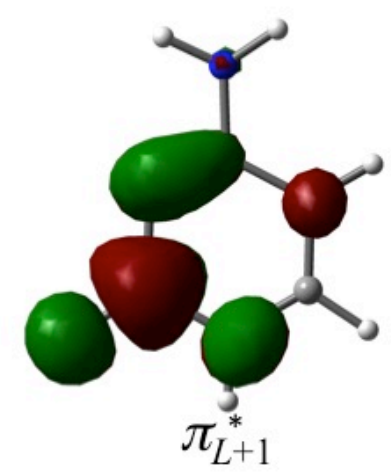

Figure 3. Orbitals involved in the vertical transitions of cytosine calculated in vacuum at the $\operatorname{CASSCF}(14,10) / \mathrm{cc}-p$ VTZ level of theory. 


\section{For Table of Contents use only}

Polarizable QM/MM multi-configuration self-consistent field approach with state-specific corrections: environment effects on cytosine absorption spectrum

Quansong Li, Benedetta Mennucci, Michael A. Robb, Lluís Blancafort, Carles Curutchet
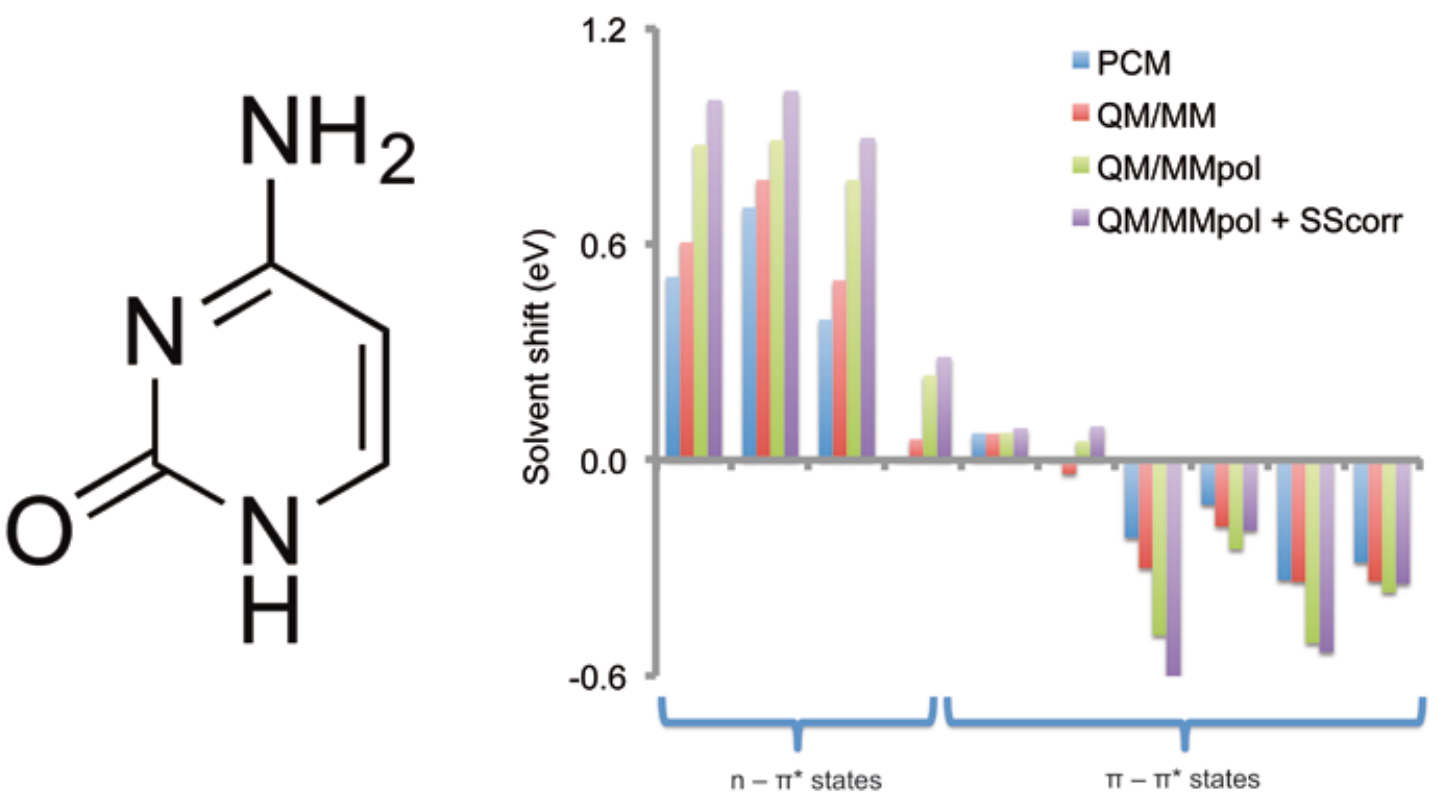